\title{
El perfeccionamiento docente en Chile (1990-2007): ¿Estado versus mercado?*
}

\author{
Sebastián Donoso Díaz
}

Universidad Talca (Chile), Instituto de Investigación y Desarrollo Educacional

\section{Introducción}

El texto analiza las políticas explícita e implícitas (silencios) de la formación continua de los docentes del sistema educacional público chileno, impulsadas desde el año de 1990 en adelante, hito que marca el retorno de la democracia en el país y la intencionalidad de gobiernos de corregir los fallos del mercado tras la imposición hacia fines de la década del 1970 de un ortodoxo modelo neoliberal a todos los ámbitos de la sociedad chilena.

El trabajo se desarrolla desde la certeza que la profunda crisis que afecta al sistema educacional chi-

"Este texto se escribió en el 2007 como profesor visitante en la Universidad de Valencia (España). Una versión preliminar de este trabajo se presentó en Seminario sobre Política Educativa en Chile. Programa de Investigación en Educación de la Universidad de Chile, Fondo de las Naciones Unidas para la Infancia (UNICEF), Santiago noviembre de 2006. Se agradecen los comentarios de Cristián Bellei, Andrés Donoso R., María Angélica Oliva y Claudio Oyarzún. En el texto se emplean indistintamente como sinónimos: docente, profesor y maestro. leno - que irrumpió incontrolablemente en mayo del año 2006 - atañe también el ámbito de la formación continua y ésta no puede tratarse como un fenómeno desligado del episodio señalado. Por el contrario, ella es tributaria de la radical reforma del sistema educativo del año de 1981.

El retorno de la democracia en Chile coincide con el renacer de la esperanza en la educación como instrumento eficiente para alcanzar el progreso: en los países desarrollados y en las experiencias exitosas de la llamada "industrialización tardía" en otras latitudes existe un claro reconocimiento del carácter central que tiene la educación y la producción del conocimiento en el proceso de desarrollo, y en los países de la región esta actitud se ha extendido progresivamente (CEPAL/ UNESCO, 1992, p. 3).

Se trata de una perspectiva con un ilimitado optimismo acerca del papel de la educación en la sociedad (Tedesco, 1998, p. 6), fenómeno vivido ya en iguales términos en la década de los 1960, con el auge de la teoría del capital humano.

En la última década del siglo XX, las expectativas sobre la educación llevaron el gobierno a fortalecer el 
trabajo docente, implementando estrategias de perfeccionamiento profesional orientadas a lo instrumental, lo formativo, como a la especialización avanzada. De igual forma se operó con modalidades de trabajo escalar (grupos que van capacitando a otros) dirigidas a sectores específicos como también de cobertura masiva. Estos procesos buscaron incrementar la eficiencia técnica como de igual forma redignificar la profesión tras la fuerte pérdida de su valor social acaecida en la década de los 1980 .

La organización del perfeccionamiento en Chile ha sido diversa, desde talleres de autoperfeccionamiento, pasando por cursos formales específicos, generales, de profundización para empoderar la reforma en los maestros, pasantías de profesores en el país y en el extranjero, generación de una red de maestros, programas presenciales de especialización en áreas-claves etc., finalmente producto de los resultados de la primera etapa de la evaluación docente realizada el año 2005 , se han implementado talleres de desarrollo docente (obligatorios) para maestros que fueron evaluados en la categoría deficiente.

El artículo identifica los principales problemas que conforman esta temática, demandando la comprensión de los cambios que incidieron en el ejercicio docente con la implantación de la "Reforma" del Sistema Educativo del año de 1981 - impulsada en plena dictadura cívico/militar - y entender los pasos seguidos desde el año de 1990 hasta nuestros días por las instituciones dedicadas a la formación y perfeccionamiento de los maestros. Sintetizando estos aspectos en algunos desafíos que se vislumbran.

\section{Contextualización del perfeccionamiento docente: Estado, mercado educativo y laboral docente, currículo escolar}

El papel estratégico que consensuadamente se otorga a los maestros en el éxito de las políticas educacionales no ha sido consistente con las políticas e iniciativas implementadas por el Estado chileno en este ámbito, las que no han tenido la prioridad requerida en los procesos de reforma y cambio educativo, evidenciando que el país ha resuelto de manera insatisfactoria esta temática.
Conceptualmente, el perfeccionamiento docente incluye tanto la formación inicial de profesores como la actualización profesional. Dado que son herramientas políticas diferentes que responden a objetivos alternativos, requieren análisis diferenciados. En Chile son vistos como dos aspectos complementarios aunque propios. La formación inicial corresponde al diseño de la profesión en su sentido amplio, busca desplegar las competencias para el desempeño de la profesión. El perfeccionamiento - tradicionalmente ha sido establecido para alcanzar un impacto más próximo y está remitido a competencias específicas, actualizaciones o desarrollo de nuevas competencias, profesionales.

Comprender el rol sistémico que ocupa el perfeccionamiento implica considerar las exigencias y presiones que inciden en el desempeño profesional de los maestros: las demandas de la sociedad, el Estado, el mercado educativo, el mercado laboral docente y el currículo escolar. Estos elementos involucran las características laborales de los docentes para desempeñarse en los establecimientos escolares. Aunque las demandas sociales sobre los maestros se expresan heterogéneamente, es en relación de su eficiencia técnica donde la familia y los principales actores del sistema social esperan importantes mejorías que impacten positivamente en los aprendizajes de los estudiantes.

Estas demandas envuelven cambios en el servicio educativo, en la responsabilidad de los profesores, y también en el accionar del Estado. El rol subsidiario del Estado chileno asumido desde mediados de la década de los 1970 a la fecha ha generado severas limitantes en la forma de cautelar su misión como garante de la calidad de la educación, debido a que aún no dispone de un soporte legal adecuado para cumplir ese objetivo. ${ }^{1}$ En la actualidad no hay meca-

${ }^{1}$ En el informe final del Consejo Asesor Presidencial para la Calidad de la Educación (diciembre 2006) se analiza el tema y se formulan sugerencias en esa dirección, aunque hubo diferencias, el fortalecimiento del papel del Estado en la fiscalización y acreditación de los empresarios educacionales alcanzó elevado acuerdo, lo que no implica un cambio inmediato de la situación. 
nismos eficientes, legales ni financieros para articular e integrar al poderoso sector empresarial particular que provee educación pública, en una política común y compartida con los agentes estatales, que permita el funcionamiento de una carrera docente que vincule la formación, perfeccionamiento y progresión en la profesión. Como señala Pavez (2003), es difícil justificar que los regímenes laborales y de remuneraciones de los maestros que se financian con el mismo tipo de subsidio público sean tan desiguales según corresponda el empleador a un agente privado o público.

Sistemas educativos estructurados bajo principios operacionales diferentes, a saber el de Holanda, Finlandia, Suecia, Noruega, Nueva Zelanda e Australia, tienen sólidamente vinculado el perfeccionamiento de los maestros con la carrera docente, la operación del mercado de trabajo del sector, el financiamiento del sistema y las demandas del currículo escolar. En Chile esta situación no presenta una sinergia equivalente. Se entiende que el mercado se regula solo, por ende hay independencia de los factores señalados, lo que introduce ineficiencia al generar una fuerte asincronía sistémica, pues los componentes no se relacionan, adicionando complejidad a la resolución de esta problemática. No existe un vínculo formal (más que el título habilitante) entre el diseño de una carrera profesional que combine perfeccionamiento y desempeño con la formación inicial. Este es un problema estratégico que afecta el funcionamiento del sistema en su sentido más amplio.

El cambio de rol del Estado tras el golpe militar de 1973 impactó al sector educación en los años de 1980 en tres aspectos centrales: en el traspaso de la gestión de los establecimientos escolares desde la dependencia del gobierno central a una descentralizada, la municipal (existiendo 345 municipios en el país). Segundo, la transferencia de los docentes de funcionarios públicos a funcionarios municipales, precarizando el mercado de trabajo; y tercero, el cambio del sistema de financiamiento de la educación pública, desde un modelo basado en la oferta a uno sustentado en la demanda, estimulando la incorporación masiva de agentes privados al mercado de la educación.
Las huellas de esas medidas en este cuarto de siglo siguen manifestándose. El sector de establecimientos privados que reciben financiamiento público casi han triplicado su participación en la matrícula escolar, mientras que las instituciones públicas la han reducido del 80 al 50\% (MINEDUC, 2005). En la actualidad el mercado escolar funciona con dos grandes tipos de operadores: los municipales y los particulares subvencionados, al que se suma el sector pagado y las corporaciones con algo más de un $10 \%$ de participación.

Las implicancias de lo señalado sobre el mercado laboral docente se reconocen en diversos trabajos (Mizala \& Romaguera, 2003; Rama \& Navarro, 2004; Rojas et al., 2006), compartiendo el argumento que el mercado educativo es un conjunto de submercados o mercados diferenciados, más que un solo mercado. Se trata de realidades acotadas que tienen a funcionar entrópicamente con ciertas reglas de mercado, pero con adecuaciones importantes. Las barreras de ingreso de nuevos docentes al sistema son bajas. La exigencia de acreditación de eficiencia profesional (calidad) por el profesor se remite a la certificación del título profesional $\mathrm{y}$, fundamentalmente, responde a la decisión de disponibilidad de recursos financieros del establecimiento (plaza) para contratarle.

Adicionalmente, la movilidad laboral transversal en el mercado (cambio de institución) también es baja. Los maestros privilegian estabilidad laboral versus el aumento de sus ingresos económicos (Mizala \& Romaguera, 2003, p. 549). Por su parte, la movilidad horizontal es aún menor, no existe un sistema organizado de carrera funcionaria, regional o nacional, lo que reduce la posibilidad de articular criterios comunes en este plano. Incluso en referencia a los cargos directivos, la nueva ley de renovación de directivos del ámbito municipal que debió aplicarse masivamente a partir del año de 2006, no tuvo el soporte financiero requerido para operar conforme fue definida.

Cada submercado funciona con criterios propios de selección de los docentes (según sus dueños). Las instituciones privadas de tradición o de creencias religiosas o filosóficas seleccionan docentes - por lo ge- 
neral - a partir de aspectos formales relacionados con la universidad donde se graduó. En escasas ocasiones se emplean desempeños demostrativos, lo que confirma que este proceso no se correlaciona directamente con las exigencias de ejecución de la función docente (Rojas et al., 2006). Este es un fenómeno arraigado en la "práctica cultural docente". El sistema de evaluación docente instaurado en Chile a partir del año de 2006 se basa en un portafolio con énfasis en tareas de planificación más que en la ejecución (consulta la visita a una sesión presencial previo aviso al docente ${ }^{2}$ ), confirmando las dificultades de coherencia entre los criterios de eficiencia técnica del desempeño docente con los empleados para la selección y evaluación de los profesores.

Esta situación ha sido caracterizada por el BID como la vigencia de dos clases de políticas educativas: unas referidas al mejoramiento de calidad y eficiencia que son rígidas y resistentes a todo cambio (las de remuneraciones e incentivos de los maestros); y otras de tipo periférico, orientadas a la expansión y crecimiento de la matrícula, que son adaptables y hasta volátiles (BID, 2006, p. 241). Ello se debe a que la educación carece de una definición básica que, una vez formulada, confiera coherencia al conjunto del sistema, permitiéndose cambios parciales sin alterar la propuesta general. Siendo este un problema estratégico, a ello se suman la complejidad para identificar la relación entre "el esfuerzo del docente" con impacto en "el producto final: el aprendizaje y la educación". Resultado que además se presenta desfasado en el tiempo, aumentando la complejidad de la temática.

Los problemas estratégicos mencionados, a saber: la falta de sinergia entre los diversos componentes y la carencia de una definición básica que confiera a la educación coherencia con los instrumentos para su implantación, en el caso chileno, se potencian con las características del Estado neoliberal. El diseño de la institucionalidad sectorial de educación no permite

${ }^{2}$ Ello se agudiza cuando son reevaluados aquellos maestros calificados como "deficientes", donde al año siguiente el portafolio aumenta su peso sobre la evaluación final. articular a los responsables nacionales del sector (ministerio) con los responsables locales: los municipios. Se usa un diseño matricial que no ajusta: lo que los transforma en nudo crítico clave. Este "error" de diseño, que permanece vigente, ha sido denunciado con antelación (Donoso \& Schmal, 2002; Donoso, 2005), y es gracias al Informe del Consejo Asesor (2006) que el país comprende su contrasentido: la matriz "Ministerio-Municipio" no tiene mecanismos eficientes de compatibilidad, lo que muestra una contradicción de proporciones. Siendo concordante con la visión de Estado subsidiario, el ministerio no puede generar alianzas estratégicas con socios estables en los distintos procesos que debe afrontar, en especial en los de formación continua (Beca, Montt \& Sotomayor, 2006, p. 30).

Los elementos señalados introducen incertidumbre e imponen al currículo y prácticas pedagógicas un rol estratégico en este campo. Se entiende que el currículo determina criterios de desempeño y a partir de ello se establece la brecha de perfeccionamiento de los maestros (la diferencia entre sus prácticas habituales y las del nuevo currículo). Esta dimensión del problema es más compleja de mitigar. Implica introducirse en el "aprendizaje profesional" modelado tras años de trabajo en el establecimiento escolar. Los cambios en las prácticas docentes se enfrentan a un hacer histórico, enraizado en rutinas que "incorporan" las nuevas propuestas curriculares como variaciones de lo mismo, sea por la dificultad de identificación real que implican, como por que "la manera de funcionar (las prácticas) de los centros escolares forman (y deforman) a los docentes en su ejercicio profesional" (Vaillant, 2004). ${ }^{3}$

La situación reseñada es expuesta por Navarro (2002, 2006) como una debilidad del Estado para generar políticas intemporales, las que redundan en que los gremios docentes logran establecer rigideces al sistema de estabilidad laboral, afectando los sistemas

${ }^{3}$ Un refrán dice que todas las reformas educativas mueren en la puerta del salón de clases. Esta temática es reconocida plenamente por muchos autores (José Gimeno Sacristán, entre otros). 
de promoción y evaluación, el funcionamiento del mercado de trabajo y, por esta vía, los mecanismos orientados a vincular eficiencia técnica, desempeño profesional y perfeccionamiento docente en la carrera docente. Un problema severo de eficiencia ${ }^{4}$ que deben asumir los sistemas de remuneraciones docentes es cómo asociar remuneraciones a incentivos de desempeño en escenarios políticos en que el Estado no puede imponer - sino negociar - estos aspectos (Liang, 2003; Mizala \& Romaguera, 2004).

\section{Perfeccionamiento docente y "hábitos profesionales y gremiales"}

En el caso chileno,

\section{[...] la constitución de la profesión docente está estrecha- mente asociada al llamado Estado Docente, es decir, a la notable y persistente intervención del Estado nacional en la organización y desarrollo del sistema educacional chileno desde la independencia en adelante. (Núñez, 2003, p. 455)}

Por ello los cambios de 1981 impactaron hondamente en la "seguridad" de los docentes como funcionarios públicos, ya que no se autoconcebían - por entonces - desde otra perspectiva que aquella.

El siglo XX concentra el auge y caída de los maestros desde la perspectiva social y profesional. Los principales movimientos culturales, sociales y políticos de la primera mitad del siglo recién pasado tienen a los profesores entre sus militantes más destacados (Vial, 1987). Los congresos pedagógicos de comienzos del siglo XX muestran la visión nacional y las políticas de Estado en educación y en el ámbito social propuestas por los maestros (Núñez, 1997). Este rol fue perdiéndose en el tiempo. La pauperización social de la profesión docente redujo su capital cultural (Cruz, 2002; Adler \& Melnick, 1998). En forma paralela se desarrolló una fuerte cultural gremial, configurada por subculturas derivadas del ordenamiento

${ }^{4}$ Se entiende eficiencia como el funcionamiento en equilibrio del mercado sin distorsiones. horizontal como vertical del sistema educacional: los profesores normalistas, los profesores de Estado etc. como en sentido vertical, los profesores católicos, los masones etc. (Núñez, 2003), generando una red de vínculos sociales que, a su vez muestran una práctica muy propia de transacción de relaciones (Adler \& Melnick, 1998).

La vida gremial y profesional está fuertemente imbricada en el movimiento docente. Hasta 1973 existía el Sindicato Único de los Trabajadores de la Educación (SUTE), que agrupó a maestros, paradocentes y al personal que trabajaba en el sector, mostrando una fuerte visión gremial que es fracturada por la dictadura y que se recompone - más de una década después - con la creación del Colegio de Profesores (restringida a quienes ejercen la docencia). Tras las primeras elecciones democráticas de sus autoridades, el colegio retoma parte de su visión sindical/reivindicativa, dando cuenta con ello del arraigo histórico de la práctica gremial. Ello significó que paulatinamente se vaya asumiendo una propuesta de defensa gremial profesional (Pavez, 2003, p. 78; Colegio de Profesores, 2006), imprimiendo más fuerza a la discusión de las tareas del sector, como fue el Congreso Pedagógico de 2005, siempre con sesgo gremial. A este proceso contribuyeron decisivamente las prácticas docentes impulsadas por la reforma educativa de 1965 y la "reorganización curricular" de la dictadura, que fomentan la visión técnico/operacional de la pedagogía, fortalecida por el enfoque conductista en la formación docente.

Este fenómeno es paralelo al de otros países de América Latina. La pauperización socioeconómica y cultural de los docentes (Vaillant, 2004, p. 11), el envejecimiento de los docentes (Navarro, 2002, p. 25; Núñez, 2003, p. 516), la feminización de la profesión (Liang, 2003), que en Chile es muy amplia en educación básica y menor en enseñanza media (Núñez, 2003, p. 516); y un envejecimiento significativo del profesorado que trabaja en el sector público (idem, p. 515-516): estos factores inciden en una depreciada valoración social de la profesión docente, situación revertida en parte en los últimos años gracias al 
incremento de sus remuneraciones, el desarrollo de becas para estudiar pedagogía y otras iniciativas. Pese a ello persiste el problema, requiriendo de una estrategia que involucre factores como las condiciones de desempeño, las características del mercado de trabajo docente, la estructura de remuneraciones, el sistema de formación inicial y continuo etc. (Consejo Asesor..., 2006, p. 161 -164).

Como se expuso, la reforma de los años de 1990 presentó como un eje clave "la profesionalización docente", no sólo como respuesta a presiones del magisterio que buscaba revertir el proceso de municipalización y de descentralización y su impacto en las condiciones laborales y salariales de los profesores, situación que parecía - y aún parece - casi irreversible. La ley del Estatuto Docente promulgada en 1991

\section{[...] fue una respuesta a necesidades políticas propias de la etapa de transición a la democracia. No podía desoírse las demandas gremiales de contar con una legislación específica que diese estabilidad y reconociese derechos y garantías per- didos. También era necesario hacerse cargo de la desafección de los docentes hacia la descentralización privatizante y su expectativa de recobrar su estatus de funcionarios públicos. \\ (Núñez, 1999, p. 180)}

El estatuto y sus correcciones siguientes establecen un ingreso económico básico común, algunas normas laborales compartidas y cierta flexibilidad en el sector municipal para determinar la planta docente. Instrumentos insuficientes según la opinión mayoritaria de los consejeros reseñada en el Informe del Consejo Asesor de Educación (2006), sin embargo el documento deja algunas dudas " [....] el Consejo ha llegado a la convicción de que se requiere crear una carrera Profesional Docente en el país. No existe acuerdo si esta deberá regir también el sector de establecimientos particulares financiados por recursos del Estado" (Consejo Asesor..., 2006, p. 166). El párrafo da cuenta (i) que no existe una carrera docente; (ii) que es importante generarla; (iii) incluso el informe detallan sus virtudes, sin embargo, (iv) al ser aplicable sólo a los docentes del sector municipales mantiene la estructura de submercados, y con ello no se resuelve el problema estratégico fundamental: la falta de sinergia entre los diversos factores relacionados con formación y perfeccionamiento docente, mercado de trabajo, estructura de remuneraciones y mercado educativo.

El énfasis en la profesionalización docente como propuesta de revalorización de los profesores implica:

(a) Dominio apropiado de una competencia técnica, sobre bases de conocimiento científico y teórico alcanzables sólo en una formación de nivel superior; (b) reconocimiento de la sociedad acerca del papel de interés público que cumple la profesión y las consiguientes retribuciones materiales; (c) responsabilidad de los miembros de la profesión respecto a su desempeño en el campo que la sociedad les confía, y (d) autonomía en el ejercicio de la función (República de Chile, Secretaría General de la Presidencia. (apud Núñez, 2003, p. 477)

Estas orientaciones son coherentes con los procesos de profesionalización de otros países y muestran más que especificidad del trabajo educativo, elevada comunalidad con su definición en otros contextos sociales (Schulmeyer, 2004, p. 27-30).

El desarrollo profesional docente es definido como "el proceso continuo de crecimiento de las competencias para la enseñanza, desde la formación inicial hasta el fin de la carrera profesional" (Beca et al., 2006, p. 14). Con anterioridad se había señalado que las dimensiones claves para el desarrollo profesional son: "Formación inicial, perfeccionamiento continuo, condiciones de trabajo en las escuelas y liceos, y la institucional ministerial necesaria para implementar un sistema que apoye con eficiencia el desarrollo de la profesión" (idem, ibidem). En tal sentido estos procesos asumen como componente clave la formación inicial y su carácter continuo. Ello se aprecia en el texto del Ministerio de Educación sobre el tema (idem) y en el informe del Consejo Asesor (2006, p. 163175). En ambos la formación inicial es el componente central de la estrategia de profesionalización, dando 
cuenta de la postergación de la formación continua ante la inicial.

En los "hábitos docentes" son mejor evaluados los procesos de perfeccionamiento con componentes mayoritariamente aplicables, más próximos al plano técnico. Ello responde al derrotero que ha seguido la formación docente y más que eso, a lo que la misma práctica profesional valoriza y la sociedad retribuye: los resultados referidos a los aprendizaje de los estudiantes (ISUC, 2005; Donoso, Cancino \& Vidal, 2006). Sostenedores públicos y privados enfrentan desigualmente este proceso, algunos responden a una política institucional de inversión, usualmente los de mayor data o que atienden la población de mayor capital social y cultural, habiendo otros que la ven como una inversión del docente para mantener su empleo (Rojas et al., 2006). Ello sintetiza otro aspecto estratégico: la falta de visión compartida del sentido y rol del perfeccionamiento en la profesionalización y, por ende, del impacto en los resultados educacionales.

\section{La formación de los profesores en Chile: 1980-2005}

La reforma del año de 1981, que también involucró a la educación superior, mostró escasa visión estratégica de sus diseñadores en materia de políticas formación inicial de docentes y, ciertamente, una desafección por las consecuencias de estas decisiones en el mediano y largo lazo. Esta reforma, sin mediar estudio evaluativo alguno le restó a la formación de profesores el rango de profesión exclusivamente universitaria. De esta manera, la formación inicial de maestros perdió la prerrogativa de carrera universitaria, pudiendo ser dictada también por los institutos profesionales, reduciendo por ello significación y valoración social, a lo que debe sumarse el severo castigo que registraron los docentes en sus remuneraciones en esos años.

Durante los 1960 y primeros años de los 1970 la tendencia seguida por las remuneraciones de los docentes no fue muy distinta de la exhibida por el promedio de la economía.
Sin embargo, a partir de 1974 se produce una desviación entre la evolución de los sueldos de los docentes y la del promedio de la economía. En particular, las remuneraciones reales del promedio de la economía aumentaron un $21 \%$ en 1974 respecto de 1973, mientras las del profesorado cayeron un $50 \%$ en igual período. En tanto, en la década de los 1980 se habría producido nuevamente una divergencia importante, que habría llevado a que las remuneraciones de los docentes cayeran un 38\% en el periodo 1981-1990 mientras las del promedio de la economía sólo lo hicieron un 3,8\% . [...] esta situación habría ubicado las remuneraciones del profesorado en 1990 a niveles no sólo inferiores a los exhibidos durante los primeros años de los 70 y 80 sino que por debajo de los observados durante 1960. (Rojas, 1998, p. 168-169)

La reforma redujo significativamente las atribuciones del Ministerio de Educación para fiscalizar la educación superior. Por otra parte, las señales de las autoridades nacionales y sectoriales y también las del mercado (incluyendo el laboral) hacia la formación de maestros eran negativas. Ello significó pérdida del valor de la educación y se tradujo en la década de los 1980 en una fuerte contracción presupuestaria que implicó reducir el presupuesto en un $25 \%$ real en ese período y bajar la participación del sector público en el PIB de 17\% correspondiente al año de 1973 al 2,4\% en 1990 (Cox, 2003, p. 45-46).

Las consecuencias sobre las demandas de vacantes pedagógicas fueron fuertes. Algunas universidades cerraron - temporal o definitivamente - su área pedagógica. Durante varios años casi todas las universidades que seguían dictando pedagogías no llenaron sus cupos. Preferente las universidades tradicionales privadas, confesionales y laicas, permanecieron con esas carreras abiertas, aunque con fuerte reducción presupuestaria. Por su parte, nuevas universidades privadas e institutos profesionales ofrecieron carreras del área, ayudadas por su bajo costo operacional.

En los años de 1980 los problemas en la formación de maestros, en especial de enseñanza básica, se acrecentaron con la vigencia de titulaciones extraordinarias de docentes que estaban en ejercicio. La masividad de estos programas de formación, casi sin 
control público, generó gran heterogeneidad formativa y permitió que algunas universidades les instalaran como programas "extraordinarios permanentes" de formación - vigentes hasta el año 2006 - cuyas características fundamentales son: a) requisitos de admisión no equivalente al pregrado regular; b) períodos lectivos menores o procesos concentrados que implican gran esfuerzo de asimilación; c) ofrecidos en locales sin el adecuado soporte académico, infraestructura y equipamiento; d) en oportunidades implicó competencia desleal con sus mismos programas de pregrado (menor tiempo de estudio o de dedicación, costos diferentes etc.); e) estos programas se transformaron en estratégicos ara la supervivencia financiero/institucional de algunas universidades.

Las conclusiones de un estudio sobre el tema son elocuentes:

A la luz de los resultados arrojados por el presente estudio, consideramos que las "razonables dudas" acerca de la calidad de estos programas, expresados tanto por la Comisión sobre Formación Inicial Docente como por el ex-Ministro son fundadas, con un nivel suficiente de detalle en este estudio, y efectivamente se constituyen en un riesgo grave para la educación de nuestro país, ciertamente no se garantiza una formación de calidad de acuerdo a los estándares necesarios. (Rufinelli \& Sepúlveda, 2005, p. 49)

Por su parte, el Colegio de Profesores en su Congreso del año de 2005 denuncia "la existencia de instituciones generadoras de mala calidad académica, que no cuentan con parámetros mínimos en la formación de un profesor, lo que se agrava por la escasa o nula fiscalización de parte de la autoridad" (Colegio de Profesores, 2005).

La nueva ley de acreditación de carreras y programas académicos, promulgada recientemente (2006), plantea como obligatorio que tanto medicina y pedagogía se acrediten bajo normas especiales. Para esta última debe hacerse desde el primer año de funcionamiento y debiendo existir una examinación externa de sus egresados con carácter decisivo para su ejercicio profesional.

\section{El perfeccionamiento docente: 1990-2005}

Al tenor de los resultados del sistema educativo (2006) es razonable preguntarse: ¿por qué no existió una política de Estado en materia de perfeccionamiento docente en esos años? Es posible que fuese por las tareas urgentes y complejas de recuperación del deteriorado sistema educativo nacional postdictadura, lo que explicaría que quedase postergada para una etapa más avanzada, pero es difícil extender este argumento para un periodo de quince años, más aún cuando los docentes son una parte muy significativa del problema que se buscada revertir.

\footnotetext{
¿De dónde se parte? La educación chilena al inicio del Gobierno del Presidente Aylwin exhibía logros de importancia en la cobertura, el aumento en la escolaridad promedio de los chilenos y la reducción del analfabetismo. En contraste, el sistema educativo mostraba indicadores críticos en cuanto a la calidad de la educación y a la equidad de su distribución. También había fuertes problemas de gestión, ya que la situación de los profesores y los niveles de financiamiento de la educación se habían deteriorado mucho durante la década de los 80. (García Huidobro, 1999, p. 9)
}

Es por ello que Núñez (2003), analizando las políticas de profesionalización docente, señala:

Los objetivos y los logros de la política estatal de "fortalecimiento de la profesión docente" han sido de instalación gradual, no exentos de dificultades o errores específicos de gestión, además de carentes de suficiente participación social, sea en al base del sistema o en su cúpula decisional. (p. 488)

Las lecciones de este proceso se refieren al ajuste entre los pasos para alcanzar éxito en la implantación de las políticas versus deseos y voluntades por forzar su puesta en escena, que de no respetarse los primeros terminan debilitando los segundos. Núñez da cuenta que los docentes no tuvieron un rol protagónico en el diseño de la reforma, pese a que se les reconocía y necesitaba para su éxito. A partir del año de 1990 las 
orientaciones educacionales hablan de la centralidad de la escuela en la política educativa chilena y del acento en los aprendizajes cognitivos y socio-afectivos (Sotomayor, 1999, p. 75). "El corazón de esta reforma es el logro de más y mejores aprendizajes intelectuales y morales para todos. Este 'corazón' está en la escuela" (Discurso del Ministro de Educación, inauguración del año escolar de 1997, apud García Huidobro \& Sotomayor, 2003, p. 253). Ese aprendizaje y la centralidad de la escuela están ligados al hacer docente, de allí lo incomprensible del vacío (silencio) de las políticas de perfeccionamiento hacia los maestros.

De esta forma, más que la ausencia de una política estratégica en esta materia, se dio paso - por parte del Estado - a la implementación de diversos programas: “(el Estado) no llega a formular una política integrada y coherente de formación y desarrollo profesional docente para el mediano plazo. [...] Las acciones para docentes se mostraron como 'emergentes', según las demandas planteadas" (Ávalos, 2003, p. 559). Las razones que originaron respuestas programáticas a iniciativas parciales son una incógnita, en vez de la estructuración de una política de formación continúa inserta en la reforma que se proponía al país.

\section{El perfeccionamiento docente: 1990-1999}

En esta década no existió una política pública de perfeccionamiento más allá del Estatuto Docente. En consecuencia, no era dable esperar sincronía entre las políticas educativas con el papel desempeñado por la formación continua. De igual forma, la propuesta del Banco Mundial, agente financiero de las reformas educativas de los años de 1990, no asumía como componente sistémico el perfeccionamiento de los maestros sino como factor asociado a políticas específicas. Esta situación es consistente con la visión tecnocrática de reforma educativa, donde se asume la participación de los docentes como una dificultad para la implantación de las políticas, reduciéndosela a instancias puntuales (Gysling, 2003) a saber: análisis de la propuesta curricular de la enseñanza media (Donoso, 2005) y la evaluación de la jornada escolar completa (2006), que no son instancias de validación de políticas, sino de información acerca de éstas.

La ausencia de una participación institucionalizada de los docentes en el diseño de la reforma podría interpretarse como de desconfianza o de prescindencia (lo que también refuerza la visión tecnocrática). Esto cambia con la discusión del sistema de la evaluación docente, desde el año de 2005 en adelante, fruto de un paciente proceso de aunar voluntades y crear consensos para lograr el objetivo (Ávalos \& Assael, 2006). El rol terciario de las políticas de perfeccionamiento en la década se sustenta en dos indicadores: a) no hay documentos oficiales de políticas sobre la materia, excepto algunos elementos consignados en el informe de la Comité Técnico Asesor del Diálogo Nacional sobre Modernización de la Educación (1994); y b) la unidad ministerial encargada de este tema; el Centro de Perfeccionamiento, Experimentación e Investigaciones Pedagógicas (CPEIP) asume un rol confuso, asiéndose a su función como administrador nacional del perfeccionamiento de los maestros.

Durante la década en análisis el perfeccionamiento se insertó en los programas focalizados impulsados centralizadamente por el ministerio, que no estaban integrados a la estructura tradicional, donde su orientación era funcional al logro de las metas. La iniciativa pionera fue el programa de escuelas focalizadas (P900), dirigido a trabajar con los establecimientos escolares con los más bajos resultados en las pruebas (Sistema Nacional de Medición de la Calidad de la Educación - SIMCE ${ }^{5}$ ). El P900 planteó los Talleres docentes, para familiarizar a los maestros con los materiales e incorporar las nuevas propuestas pedagógicas al aula (Sotomayor, 1999). Este programa tuvo un período muy exitoso (Dipres, 2001a, p. 7; OCDE, 2004; MINEDUC, 2000) pese a lo cual el año de 2002 fue reorientado, con la finalidad de instalarlo en el accionar regular del ministerio y

\footnotetext{
${ }^{5}$ El SIMCE consiste en pruebas censales aplicadas anualmen-
} te a los alumnos de $4^{\mathrm{a}}$ grado y año por medio a los 8 y 12 grados. Las pruebas son en lenguaje, matemática, ciencias naturales y ciencias sociales. 
romper el efecto de "no egreso" de muchos establecimientos escolares que permanecían una larga data en el sistema. ${ }^{6}$ Como ocurre con los impactos de este tipo de programas, es muy difícil aislar el efecto de un componente sobre otros y donde inicialmente no se estableció una línea de base para contrastar los resultados. Su rediseño lo transformó en el Programa de Escuelas Focalizadas, manteniendo el debate sobre este tipo de estrategias.

El Programa Mece-Básica fue un componente de la estrategia política del ministerio. Sus dos unidades claves: el programa urbano y el rural poseían diferencias sustantivas. El primero aportaba esencialmente infraestructura, equipamiento escolar, salud (física) de los estudiantes y desarrollo de estrategias pedagógicas en la escuela mediante proyectos de mejoramiento educativo (PME). El segundo, aplicado en el sector rural, se refería al currículo de las escuelas pequeñas multigrado (uni, bi y tridocente), atendiendo las prácticas docentes de los maestros, dado que estas escuelas tenían históricamente débiles resultados escolares y población de bajo capital social y cultural. Los resultados del programa fueron muy positivos (San Miguel, 1999, p. 108; MINEDUC, 1999), quizás la transformación de mayor significación fue el trabajo en Microcentros de Profesores (con reuniones periódicas entre profesores de un territorio), buscando cambiar las prácticas de los maestros más postergados del sistema, proceso que ha continuado en algunas localidades.

El programa Enlaces, de informática educativa, asumió un proceso de capacitación de los docentes funcional a sus objetivos. Éste ha seguido el derrotero de otras experiencias internacionales equivalentes:

Las TIC no se han incorporado significativamente a los procesos de enseñanza, que los docentes la emplean como complemento pero que no forman parte del eje estratégico de su docencia; y que aunque el acceso computacional se

${ }^{6}$ Este efecto no se ha logrado completamente, siendo debatido cada vez que se entregan los resultados de las pruebas SIMCE, donde se actualiza la disputa acerca de los logros de los establecimientos "egresados" del programa. ha generalizado en la población su uso frecuente no es para fines pedagógicos. (Cancino \& Donoso, 2004, p. 151)

Esto se ha traducido en una oferta educativa insuficiente, en problemas de uso y mantención del equipamiento y en otros aspectos que alertan de los logros, incluso de parte de sus mismos gestores, quienes al reflexionar sobre los resultados de algunas investigaciones internacionales en este campo destacan su pertinencia con nuestra realidad:

a) la mayoría de los profesores utilizan las TICs para mejorar su gestión docente y, b) la mayoría de los profesores no transforma sustancialmente su práctica docente al integrar tecnologías en el aula, lo que hace es acomodar la tecnología a su práctica actual. (Hepp, 2003, p. 432)

En la enseñanza media se impulsaron dos iniciativas de distinto orden de magnitud. La primera desde el año de 1995, producto de los estudios sectoriales realizados por el componente Mece Media, tuvo por finalidad fortalecer la profesionalización mediante los "Grupos profesionales de trabajo", difundidos masivamente en los establecimientos educacionales (Ávalos, 1999). Considerando entre otros los principios de aprendizaje entre pares y de comunidades de aprendizaje, esta iniciativa enfrentó los problemas de dedicación horaria de los profesores y de trabajo en más de un centro escolar, lo que le restó sistematicidad e impacto a sus resultados. Esta actividad se aplicó en el nivel de aula del establecimiento.

El Proyecto Montegrande (1997-2002) fue una iniciativa ministerial destinada al mejoramiento de la enseñanza media mediante el apoyo a 51 liceos de excelencia. Consistió en desarrollar propuestas innovadoras "irradiables al sistema educativo", si bien no estaban centradas en la formación continua, aunque la consideraron. Una evaluación intermedia de este proceso expresó que

[...] algunos liceos no evidencian suficientemente la capacidad de innovación, lo cual ha significado retrasos y posibles reformulaciones para un término anticipado del proyecto; y la 
elaboración y posterior replicabilidad de un modelo de gestión descentralizada no se perfila con nitidez, por cuanto las características de los proyectos educativos, exitosos o no, obedecen más bien a condiciones del contexto de los liceos, las cuales no son necesariamente extrapolables. (Dipres, 2001b, p. 5)

No se conocen públicamente detalles de los resultados alcanzados. Históricamente, a mediados del siglo XX se impulsó en Chile por el Ministerio de Educación una iniciativa equivalente - de enseñanza secundaria - la que tuvo un discreto impacto (Núñez, 1997).

Desde el año de 1996 hasta pasado el 2000, funcionó el Programa de Pasantías al extranjero, involucrando unos 4.200 profesores hasta el año de 2000 (Cox, 2003, p. 66); cuantitativamente se trata de una experiencia más marginal, "dada la reducida cobertura del Programa (2,4\%), sus esfuerzos multiplicadores son insuficientes" (Dipres, 2000, p. 3). Su importancia radicó en que fue un aliciente de profesionalización a un gremio que había sido socialmente maltratado en los años del gobierno militar (Undurraga, 1999, p. 216). Su principal falencia fue que no tuvo un sistema post pasantía para difundir y reproducir los aprendizajes y aprovechar la inversión, evidenciando la fisura del enfoque matricial "Ministerio-Municipio", nudo no resueltos de gestión de la educación chilena.

El último y más masivo de los hitos de perfeccionamiento de este período fue el Programa de Capacitación para el Nuevo Currículo (conocido como PFF), vigente de 1997 al año de 2003. Fue la iniciativa de mayor magnitud: cubrió prácticamente a todo el profesorado del sector público ${ }^{7}$ con un programa de capacitación acorde al nivel en que trabajaban, que implicaba 70 horas (cronológicas). Su implementación se realizó en seis años, a partir del $1^{\circ}$ año de enseñanza básica, en grupos de dos niveles por año, incluyó también a docentes directivos. Los cursos

${ }^{7}$ Según cifras del CPEIP, el año de 1999 participaron 38.821 profesores, 55.412 el 2000, 54.870 el 2001,37.857 el 2002 y 1.834 el 2003, de un universo estimado de 103 mil docentes (Cox, 2003, p. 65; Beca, Montt \& Sotomayor, 2006, p. 35). se dictaron en los períodos de receso veraniego, con una semana presencial y otro tanto de seguimiento y apoyo. Se buscaba cambiar y renovar las prácticas docentes, familiarizando a los profesores con los principales elementos del nuevo currículo (de inspiración constructivista), su enfoque y las metodologías de enseñanza que éstos suponían. Esta tarea excedía las posibilidades de resultados positivos en ese período de tiempo de formación, demandando casi un "milagro" para que sus impactos transformaran decisivamente las prácticas docentes mediante un proceso tan breve (Dipres, 2002, p. 7).

El segundo aspecto vinculado a este proceso es que se pasó de una modalidad abierta en su diseño inicial (NB1), los cursos fueron cada vez más formateados por el ministerio. Se estima que este cambio antiprincipios de la misma reforma - se debió a que los resultados logrados a esa fecha no cuadraban con los esperados. Finalmente, en lo operacional, por el enfoque matricial "Ministerio-Municipio" en varias oportunidades los docentes que asistieron a los Talleres no fueron asignados por los sostenedores a los niveles para los cuales se habían preparado, restándole eficiencia este proceso.

\section{El perfeccionamiento docente: 2000-2005}

En esta etapa el CPEIP lidera parte importante del perfeccionamiento docente del Ministerio de Educación, como respuesta a un diagnóstico en el que se enumeran desafíos en este ámbito desde el nivel sistémico hasta el aula (Beca, 2006). Su objetivo estratégico es fortalecer el aprendizaje entre pares basado en comunidades de aprendizaje (Cerda \& López, 2006, p. 40). En esta modalidad se identifican iniciativas como los Talleres Comunales de Perfeccionamiento, orientados a profesores primarios cuya data se remonta a los inicios de 1991. Su objetivo era instalar un sistema de formación continua entre pares. Considera los ámbitos de responsabilidad profesional y preparación para la enseñanza. Su metodología se basa en la reflexión liderada por un docente guía. Este enfoque acusa la necesidad de apoyar y preparar en 
mejor forma a los profesores guías y validarlos ante sus pares. En segundo lugar demanda comprender a fondo las propuestas curriculares que se analizan, incluyendo los aspectos de evaluación (Álvarez, 2006).

El Programa de Pasantías Nacionales busca el intercambio de experiencias entre equipos docentes de establecimientos públicos. Involucra establecimientos que hayan tenido experiencias relevantes en este campo. Su principal logro es validar el aprendizaje de pares para mejorar las prácticas docentes. El programa se asocia al de redes pedagógicas (red de maestros de maestros) e implica reforzar el intercambio horizontal entre los participantes.

Tres programas impulsados por el CPEIP se mencionan sin analizarles: el de Educación Emocional, que operar a escala reducida - a marzo del 2006 habían participado 78 establecimientos y 1.488 docentes (Arellano \& Cerda, 2006, p. 146) - y que requiere de mayor tiempo para evidenciar resultados sustentables. El segundo es el programa de educación a distancia, debido a que trata aspectos puntuales y no se observan aportes. Se inscribieron 5.755 docentes y aprobó el 43\% de ellos (2.451 participantes hasta marzo de 2006) (idem, p. 220). El tercero es el Programa OFT Prensa que busca aportar al manejo de los objetivos fundamentales transversales. Entre los años de 2001 y 2003 se graduaron 102 maestros y en el año de 2005 estaban participando otros 196 más.

Los programas dirigidos a la apropiación curricular, externalizados vía licitación a centros universitarios formadores de maestros, implicaron la entrega de un diploma de curso avanzado o de una especialidad o mención según la intensidad del programa. Hasta marzo del año de 2006 habían participado 6.781 docentes, no se conoce su tasa de aprobación y algunos de los cursos estaban aún dictándose (idem, p. 252). Los problemas más severos reseñados en las evaluaciones es en apropiación curricular (ISUC, 2005; Donoso, Cancino \& Vidal, 2006; OCDE, 2004) se refieren a que los logros son escasos. Ello se debe, entre otros aspectos, a: a) dificultades importantes en la formación inicial de los docentes; b) poco dominio de conceptos claves de la disciplina que enseñan, lo que implica: mala formación, errores conceptuales, de preconceptos, de relaciones y por ende de las conclusiones a las que se puede llegar. Esta situación puede ser más generalizada de lo que se piensa y responde a décadas de baja inversión y de escaso interés social del país por el tema educacional, y también a varias generaciones de estudiantes de enseñanza básica, media y superior con errores fundamentales en sus procesos de formación que no han sido corregidos.

En una misma línea de discusión puede situarse la necesidad de implementar una reforma curricular versus la importancia de cambiar las condiciones en las cuales se producen los aprendizajes. Ambas discusiones exponen un tema más complejo: generar apropiación en los maestros. Desgraciadamente este tema no es enfocado debidamente. Los aportes del Colegio de Profesores en pleno debate curricular (congreso año 2005) asumen que el Estado puede imponer los términos del perfeccionamiento a todos los actores y que le corresponde financiarlo plenamente a éste, y permanece con la visión que la educación pública es abrumadoramente municipal.

La necesidad de comprender que el universo de la educación pública excede con creces el ámbito de la gestión municipal es también un fenómeno que no está reflejado en las Orientaciones de política de desarrollo profesional docente continuo del mismo Ministerio de Educación (2004). "A nivel extrasistema, interesa establecer articulación con los sostenedores municipales", extendiéndose el resto del documento - 2 páginas - en reafirmar la importancia de trabajar con los sostenedores municipales. En palabras de la ministra de Educación a febrero del 2006, se posiciona este tema como el eje de todo el proceso educativo "para asegurar a todos una educación de calidad, el desarrollo profesional de nuestros docentes es, sin lugar a dudas, la prioridad uno del esfuerzo educativo nacional" (Beca, Montt \& Sotomayor, 2006, p. 7). Si bien el documento no convoca a la alianza Ministerio de Educación-Municipios, no queda en evidencia si se trata de orientaciones para un sistema nacional de formación continua para los maestros de dependencia municipal o para cualquier docente. Los debates del Consejo Asesor (2006) en 
este plano ratificaron que el subsistema de educación particular - subvencionado y pagado - aboga por su propia estructura de funcionamiento.

En grandes cifras el perfeccionamiento docente sigue siendo un área desregulada del sistema, evidenciando con ello el problema de institucionalidad que afecta al sistema educacional chileno y, en especial, al Ministerio de Educación como garante social de la calidad educacional. Solamente una parte del perfeccionamiento está bajo la supervisión del CPEIP (se excluyen los programas de magíster y doctorado dictados por las universidades). Su labor en este período (2000-2005), descontando los cursos del programa de perfeccionamiento fundamental PPF (por su carácter puntual y único), implicaron cuantitativamente: (i) en la línea de actualización pedagógica de 1.102 participantes en el año de 2000, se proyectan 15.446 para el año de 2005. (ii) En la línea de actualización curricular se registraron 7.629 participantes en año de 2000 subiendo a 8.594 docentes para el 2005. (iii) En formación en ámbitos de gestión, de 3.400 personas el 2003 se sube a 5.400 el año de 2005 , totalizando para este último año 29.483 participantes (Beca, Montt \& Sotomayor, 2006, p. 35). Esta cifra asume la heterogeneidad de procesos de diversa intensidad, duración, modalidad etc. Si bien se puede sostener que en estos años el perfeccionamiento ha recuperado cierto protagonismo, está lejos de jugar un rol estratégico en la actualización de las competencias de los docentes.

Vista la formación continua de docentes desde la perspectiva política, Chile sigue mostrando una falencia importante en el ajuste de sus respuestas al diagnóstico del sector. La crisis de la educación nacional también afecta estos procesos. Las políticas de desregulación inherentes a la visión de mercado muestran sus dotes: un Ministerio sin la institucionalidad necesaria para responder a sus debidas funciones. Una oferta de formación continua muy heterogénea y sin parámetros de calidad que sostenga una información fidedigna para quienes están interesados en tomar decisiones, bajo impacto de la formación continua, y una carrera docente desligada de la formación permanente.
Finalmente dos aspectos requieren revisarse. Primero, el perfeccionamiento docente no está plenamente centrado y consistentemente diseñado para responder a los severos problemas de formación profesional - disciplinar y de enseñanza - que evidencian los docentes en los diagnósticos. Segundo, el perfeccionamiento sigue siendo tratado como un fenómeno desligado del funcionamiento del mercado educacional y desarticulado del mercado laboral docente y de los sistemas de evaluación y recompensa implementados en muestro medio, como también de las demandas de apropiación curricular.

\section{El debate entre políticas de Estado y políticas de mercado}

Las dificultades en la formación continua de docentes son parte de la severa crisis de la educación chilena. Sin embargo, su causa funcional más inmediata reside en el impacto de las reformas del año 1981. En especial, por los desprolijos procesos de formación inicial de docentes y las políticas de (des)profesionalización impuestas durante la dictadura cívico-militar. En segunda instancia, el cambio de rol del Estado en Educación y los procesos asociados a esa condición contribuyen decisivamente con la crisis presente. Es razón de ello, un aspecto insatisfactoriamente resuelto en el período en análisis es la formación permanente de docentes. Si bien en las orientaciones ministeriales destaca el papel estratégico de los maestros en el éxito de las políticas, ello contrasta - en la década del 1990 - con las iniciativas parciales que se aplicaron, muchas de escaso impacto (Beca, 2006, p. 35). Lo que fue consistente con la falta de una política sistémica de formación continua impulsada por el Ministerio de Educación.

Las correcciones llevadas a cabo en la presente década muestran asincronías severas entre esas iniciativas y los diagnósticos, escasa sinergia entre este componente con otros elementos claves mencionados: el mercado laboral docente, las políticas de remuneración e incentivo etc., las demandas curriculares y las demandas sociales. 
Debate de lo expuesto: crisis de

la educación y formación continua

La forma como la sociedad chilena ha asumido resignadamente el papel subsidiario del Estado es un problema en si mismo. Acorde con la política económica de mercado impuesta desde mediados de los años de 1970, ocurrieron cambios decisivos en la manera de pensar y de funcionar del sistema educativo. Las transformaciones en el diseño institucional derivan en un ministerio sectorial que no ha tenido las facultades necesarias para regular eficientemente el funcionamiento del sistema en toda su complejidad.

Ello ha significado asumir el mercado educativo como uno de carácter convencional, no siéndolo. La educación pública sigue atendiendo el 90\% de la matrícula escolar, pero en dos casi-mercados: el municipal (con sostenedores públicos) y el particular subvencionado. Cada uno funciona con ciertos criterios propios: organización, selección y promoción de los docentes, regimenes salariales etc. Las exigencias del ingreso a la carrera docente no se vinculan directamente con la formación inicial ni con su desempeño profesional. Tampoco hay relación con una carrera profesional que combine perfeccionamiento y desempeño, pues el ministerio no tiene facultades para regular el proceso atomizado en más de 300 empresas públicas regidos por el estatuto docente, y en un par de miles de sostenedores privados, regulados contractuales por otro criterio diferente (Almonacid, Luzón \& Torres, 2008).

Ciertamente la concepción subsidiaria alzada por el neoliberalismo no permite el diseño de la institucionalidad articulada del sector que relacione funcional y equilibradamente ${ }^{8}$ los responsables nacionales de la

${ }^{8}$ El equilibrio proviene de la adecuada aplicación del principio de responsabilidad/autoridad. Sin embargo, el Ministerio de Educación asume algunas responsabilidades para las que no tiene autoridad, dependiendo para ello de la voluntad de los empresarios. Un ejemplo se vivió en el perfeccionamiento para la reforma. En oportunidades empresarios enviaron a capacitarse a profesores para cursos en que posteriormente no se desempeñaron, sin que el ministerio pudiera intervenir. Asimismo, algunos empresarios educación (ministerio) con los responsables locales: municipios y sostenedores privados. Se trata de un diseño matricial que no ajusta. En lo operacional la matriz "Ministerio-Municipio" no tienen mecanismos de compatibilidad, derivando en contradicciones de proporciones. Consistente con esta visión del Estado, el ministerio no ha podido generar alianzas estratégicas con socios estables.

En segunda instancia, no existe una definición básica - aceptada mayoritariamente - de lo que se entiende por educación (más allá del Marco para la Buena Enseñanza), que confiera coherencia al conjunto del sistema. Ello facilita realizar cambios parciales sin comprender su impacto en el sistema, permitiendo que las políticas referidas al mejoramiento de calidad y eficiencia sean más resistentes al cambio (regímenes de contratación, remuneraciones e incentivos salariales de los maestros); y otras más adaptables (expansión y crecimiento de la matrícula). Estas relaciones responden al "hábito cultural" de enfocar el tema como si el centro fuesen los docentes y sus gremios. Ello facilita dividir el fenómeno educativo (siendo para algunos indivisibles) de manera que no se perciban los impactos entre los diversos componentes, lo que ha facilitado la instalación de iniciativas sectoriales que - en oportunidades - no son consistentes con las políticas generales.

El silencio en materia de una política pública de formación continua de maestros ha sido suplido deficientemente en la actual década por políticas parciales con limitaciones para generar impactos significativos en este ámbito. Ello no compensa la falta de una política de Estado en esta materia. Por el contrario, las políticas "parciales" conciben la formación continua como un proceso desligado operacional y sistémicamente del funcionamiento del sistema educativo: sea desde la formación inicial, como de los diagnósticos

públicos deben responsabilizarse de aspectos para los que no tiene autoridad. Por ejemplo, la ley define concurso para directivos - docentes no existiendo los fondos para ello, obligando a los municipios a aumentar su gasto, ya que no tiene la autoridad para negarse al concurso. 
sobre las fortalezas y debilidades de los docentes, la docencia, el funcionamiento del mercado docente etc. Tampoco se vislumbran mecanismos para relacionar sinérgicamente estos componentes, ${ }^{9}$ provocando una falta de perspectiva compartida sobre el sentido y rol de la formación continua en los principales actores institucionales públicos en términos de su incidencia en la profesionalización y por ende, de su impacto en los resultados educacionales.

La recomposición del movimiento gremial del magisterio - ocurrida en el ocaso de la dictadura implicó retomar parte de esta visión sindical/reivindicativa históricamente arraigada, lo que se tradujo en un cambio lento y paulatino hacia una profesionalización docente, factor complejo de incorporar pues esta imbricado con la heterogénea formación de los docentes, sustentada en enfoques operacionales de su hacer, algunos aprendidos con anterioridad a la dictadura, pero fortalecidos por el reduccionismo al que se llegó en la formación de los maestros en ese período. Ello aconteció en un escenario con fuertes cambios negativos en el capital social, cultural y económico de los docentes, incidiendo en una creciente depreciación de su valoración social como profesión.

Las consecuencias de lo expuesto provocan que la formación continua de los docentes en el país - en los hechos - tenga una importancia de tercer orden. No existió una adecuada incorporación de los maestros y sus entidades a esta temática. No se ha diseñado e implementado una política nacional sobre la materia: inicialmente las respuestas fueron funcionales, sin visión sistémica. Posteriormente se intentó un reposicionamiento del tema, aún muy parcial.

La oferta del ministerio no es del todo consistente con los discursos oficiales sobre el rol protagónico de los maestros. Las diversas líneas de formación man-

${ }^{9}$ Esta condición es difícil de superar en un enfoque de mercado "ortodoxo" como el vigente en Chile. Su solución requiere romper la fe "dogmática" en el mercado y asumir que uno de sus fallos en educación es la débil capacidad que muestra para relacionar estos componentes sin generar externalidades negativas importantes hacia la población que más depende de la educación pública. tienen una visión de educación pública como símil de sistema municipal, realidad que ya no es igual. Adicionalmente, las respuestas no articulan los principios sustentados con las propuestas específicas, ni tampoco buscan insertar adecuadamente la formación continua con los componentes del sistema señalados: demandas del mercado educativo, las exigencias curriculares etc. Esta situación es debidamente reseñada por Beca, Montt y Sotomayor (2006, p. 24-31 y p. 35-39), cuando señalan que algunos aspectos no resueltos de la formación continua son: escaso impacto de la formación continua; insuficiente apropiación de nuevas propuestas curriculares; asignación de perfeccionamiento sin foco en desempeño. También relacionado con las debilidades de las condiciones de trabajo se menciona: bajas remuneraciones e incentivos sin foco en desempeño, inconsistencia de incentivos al liderazgo educacional; trayectorias profesionales no articuladas a la formación continua; carga horaria con insuficiente tiempo para planificar clases; alto número de estudiantes por curso en sectores vulnerables.

\section{Estado o mercado en la política de formación continua}

La generación de una Política de Estado en Materia de Formación Inicial y Continua es consistente con el diagnóstico precedente. Sin embargo, esa propuesta implica un Estado políticamente activo como regulador y organizador del sistema docente en todo su sentido, y que detente las atribuciones para cumplir su tarea. Ello es un contrasentido en un esquema de mercado o un modelo de Estado subsidiario, en consecuencia en alguna medida se exige algo que está fuera del racional que no se quiere o puede cambiar.

El Estado ha manifestado un compromiso medido en los hechos con una educación de calidad. La sociedad en forma mayoritaria reclama para el sector educación "otro Estado". Si embargo en la demanda de una educación de calidad no está del todo asentada en la sociedad civil, en términos de un canal unívoco de expresión y acción al respecto. Tampoco está plenamente enraizada en los gremios docentes - donde se 
mezcla con reivindicaciones salariales - ni en los de estudiantes y padres, que carecen de la competencia para traducirles de demandas sentidas a requerimientos técnicos. Ello es funcional a una visión tecnocrática de la política, sin embargo, la esperanza de mejoría se confronta con una visión de mercado que no puede destruirse a si mismo para solucionar sus graves problemas. La posibilidad de generar consensos es compleja, la educación es un campo de fuertes intereses ideológicos, también de clase y por ende de poder.

El Estado chileno tiene una baja cuota de poder en este escenario. Su legitimación está en duda en términos de ofrecer respuesta a las necesidades y garantizar a los ciudadanos un sistema educativo de calidad: algo que en la actualidad es ampliamente demandado por la sociedad e insatisfactoriamente cumplido en la educación pública, fenómeno que en el formato de mercado parece irresoluble, salvo que opte - al menos inicialmente - por su autodestrucción.

\section{Referencias bibliográficas}

ADLER, Larissa; MELNICK, Ana. Neoliberalismo y clase media: el caso de los profesores de Chile. Santiago de Chile: DIBAM, 1998.

ALMONACID, Claudio; LUZÓN, Antonio; TORRES, Mónica. Cuasi mercado educacional en Chile: el discurso de los tomadores de decisión. Archivos Analíticos de Políticas Educativas, v. 16, n. 8, 2008. Disponible en: <http://epaa.asu.edu/epaa/v16n8/>. Acceso en: mayo 2008.

ÁLVAREZ, C. Profesores y profesoras guías: docentes que asumen protagonismo en las políticas de desarrollo profesional de las comunas. In: ARRELLANO, Mónica; CERDA, Ana (Eds.). Formación continua de docentes: un camino para compartir 20002005. Santiago de Chile: CPEIP, 2006. p. 67-74.

ARELLANO, Mónica; CERDA, Ana (Ed.). Formación continua de docentes: un camino para compartir 2000-2005. Santiago de Chile: CPEIP, 2006.

ÁVALOS, Beatrice. Capítulo 11. La formación de profesores y su desarrollo profesional. Prácticas Innovadoras en busca de políticas. El caso de Chile. In: COX, Cristián (Ed.). Políticas educacionales en el cambio de siglo. Santiago de Chile: Editorial Universitaria, 2003. p. 559-594.
Desarrollo docente en el contexto de la institución escolar. Los Microcentros rurales y los grupos profesionales de trabajo en Chile. Conferencia; Los maestros en América Latina: nuevas perspectiva sobre su desarrollo y desempeño, San José Costa Rica, 1999.

.; ASSAEL, Jenny. De la resistencia al cambio. Estudio sobre la experiencia chilena de construcción de un sistema de evaluación del desempeño. Santiago de Chile: UNESCO, 2006. Disponible en: <www.unesco.cl>. Acceso en: 5 oct. 2006 BECA, Carlos. Las políticas de formación continua de docentes. Avances y desafíos. In: ARELLANO, Mónica; CERDA, Ana (Eds.). Formación continua de docentes: un camino para compartir 20002005. Santiago de Chile: CPEIP, 2006. p. 19-31.

; MONTT, Pedro; SOTOMAYOR, Carmen. Hacia una política de desarrollo profesional docente. Chile: Ministerio de Educación, 2006. (Serie Bicentenario.)

BID - Banco Interamericano de Desarrollo. Capítulo 10. Dos clases de políticas Educativas. In: La política de las políticas públicas. Progreso económico y social de América Latina. 2006. Washington: BID, 2006. Disponible en: <www.iadb.or/res/ ipes/2006>. Acceso en: 5 oct. 2006

CANCINO, Victor; DONOSO, Sebastián. El programa de Informática Educativa de la Reforma Educativa Chilena: análisis crítico. Revista Interamericana de Educación, OEI, n. 36, p. 129-154, 2004. CERDA, Ana; LÓPEZ, Isabel. El grupo de aprendizaje entre pares: una posibilidad de favorecer el cambio de las prácticas cotidianas en el aula. In: ARELLANO, Mónica; CERDA, Ana (Eds.). Formación continua de docentes: un camino para compartir 2000-2005. Santiago de Chile: CPEIP, 2006. p. 33-44.

CEPAL/UNESCO. Educación y conocimiento: eje de la transformación productiva con equidad. Santiago de Chile: CEPALUNESCO, 1992.

COLEGIO DE PROFESORES. Congreso Pedagógico 2005. Tema 10. Punto 5. Santiago de Chile, 2005.

Los cambios que requiere nuestro sistema educativo para el desarrollo de la educación pública. Directorio Nacional, jul. 2006. COMITÉ TÉCNICO ASESOR del Diálogo Nacional de Modernización de la Educación Chilena. Los desafíos de la educación chilena frente al siglo XXI. Santiago de Chile: Presidencia de la República/Ministerio de Educación, 1994.

CONSEJO ASESOR Presidencial para la Calidad de la Educación. Informe Final. Santiago de Chile, dic. 2006. Disponible en: <www. presidencia.cl>. Acceso en: 15 dic. 2006 
COX, Cristián. Políticas educacionales en el cambio de siglo. Santiago de Chile: Editorial Universitaria, 2003.

CRUZ, Nicolás. El surgimiento de la educación secundaria y pública en Chile, 1843-1876. Santiago de Chile: DIBAM, 2002.

DIPRES. Estudio del Programa de Becas en el Exterior para Profesores (pasantías). Dirección de Presupuesto, Ministerio de Hacienda. Evaluación Gubernamental de Programas. Chile, 2000. Disponible en: <www.dipres.cl>. Acceso en: 15 oct. 2006. Estudio Programa de Escuelas de Sectores Pobres (P-900). Ministerio de Hacienda, Dirección de Presupuesto. Chile, 2001a. Disponible en: <www.dipres.cl/controldegestion/evaluaciondeprogramasgubernamentales>. Acceso en: 15 oct. 2006.

Estudio del Programa de Fortalecimiento de la

Formación Inicial docente. Ministerio de Educación. Dirección de Presupuesto, Ministerio de Hacienda. Evaluación Gubernamental de Programas. Chile, 2001b. Disponible en: <www.dipres.cl>. Acceso en: 15 oct. 2006.

DIPRES. Estudio del Programa de Perfeccionamiento Fundamental de Docentes. Chile: Ministerio de Hacienda, Dirección de Presupuesto, 2002. Disponible en: <www.dipres.cl>. Acceso en: 15 oct. 2006 .

DONOSO, Sebastián. Reforma y política educacional en Chile 1990-2004: el neoliberalismo en crisis. Estudios Pedagógicos, v. XXXI, n. 1, p. 113-135, 2005.

.; SCHMAL, Rodolfo. Elementos para definir una política de financiamiento de la educación pública. Pensamiento Educativo, n. 31, p. 256-282, 2002.

DONOSO, Sebastián; CANCINO, Victor; VIDAL, Roberto. Estudio descriptivo-evaluativo de los postítulos de educación matemática para docentes del $2^{a}$ ciclo de enseñanza básica. Santiago de Chile: CPEIP/OEI, 2006.

GARCÍA HUIDOBRO, Juan Eduardo. Parte I. Visión de Conjunto, Capítulo 1. La Reforma Educativa Chilena 1990-1998. Visión de Conjunto. In: (Ed.). La reforma educativa chilena.

Madrid: Popular, 1999. p. 7-50.

; SOTOMAYOR, Carmen. La centralidad de la escuela en la política educativa de los años noventa. In: COX, Cristián. (Ed.). Políticas educacionales en el cambio de siglo. Santiago de Chile: Editorial Universitaria, 2003. p. 253-316.

GYSLING, Jaqueline. Reforma curricular. Itinerario de la transformación cultural. In: COX, Cristián (Ed.). Políticas educacionales en el cambio de siglo. Santiago de Chile: Editorial Universitaria, 2003. p. 213-252.
HEPP, Pedro. Enlaces: el programa de informática educativa de la reforma educativa chilena. In: COX, Cristián (Ed.). Políticas educacionales en el cambio de siglo. Santiago de Chile: Editorial Universitaria, 2003. p. 419-451.

ISUC. Estudio evaluativo de las estrategias empeladas por las universidades en los cursos - taller del Programa de Formación Continua y sus efectos en la apropiación curricular. Santiago de Chile: Instituto de Sociología, Pontificia Universidad Católica de Chile/CPEIP, 2005.

LIANG, Xiaolang. Remuneraciones de los docentes en 12 países latinoamericanos. PREAL. Doc. de Trabajo n. 27, 2003. 30 p.

MINEDUC - Ministerio de Educación. Evaluación del Programa Educación Básica Rural 1992-1998. Santiago de Chile: MINEDUC, 1999.

Evaluación del Programa de las 900 Escuelas. Santiago de Chile: MINEDUC, 2000.

. Desarrollo profesional docente en el liceo. Santiago de Chile: MINEDUC, Programa MECE Media 1995-2000, 2001. . Anuario Estadístico 2005. Santiago de Chile: MINEDUC, 2005.

MIZALA, Alejandra; ROMAGUERA, Pilar. Regulación, incentivos y remuneraciones de los profesores en Chile. In: COX, Cristián. (Ed.). Políticas educacionales en el cambio de siglo. Santiago de Chile: Editorial Universitaria, 2003. p. 519-568.

. El Sistema Nacional de Evaluación del Desempeño Docente (SNED) en Chile. En: BID-PREAL. Maestros en América Latina: nuevas perspectivas sobre su formación y desempeño. Santiago de Chile: San Marino, 2004. p. 389-407.

NAVARRO, Juan Carlos. ¿Quienes son los maestros? Carrera e incentivos docentes en América Latina. Washington: BID, 2002.

. Dos clases de políticas educativas. La política de las políticas públicas. PREAL, Doc. de Trabajo Mª 36, 2006.

NÚÑEZ, Iván. Las políticas públicas en educación. Una mirada histórica (1925-1997). Estudios, Universidad de Talca/Instituto de Investigación y Desarrollo Educacional, n. 11, p. 34, 1997.

IV Parte. Profesionalización Docente. Capítulo 9.

Políticas hacia el magisterio. In: GARCÍA HUIDOBRO, Juan Eduardo (Ed.). La reforma educativa chilena. Madrid: Popular, 1999. p. 177-192.

. El profesorado, su gremio y la reforma de los noventa: presiones de cambio y evolución de la cultura docente. In: COX, Cristián (Ed.). Políticas educacionales en el cambio de siglo. Santiago de Chile: Editorial Universitaria, 2003. p. 455-517. 
OCDE. Chile. Revisión de las políticas Nacionales de Educación. Paris/Santiago de Chile: Organización para la Cooperación y el Desarrollo/MINEDUC, 2004.

PAVEZ, Jorge. Situación de los docentes, sus condiciones laborales y profesionales. In: HEVIA, R. (Ed.). La educación en Chile hoy. Santiago de Chile: Ediciones Universidad Diego Portales, 2003. p. 77-86. ROJAS, Patricioi. Remuneraciones de los profesores en Chile. Estudios Públicos, Santiago de Chile: Centro de Estudios Públicos, n. 71, p. 122-175, 1998.

ROJAS, Araceli; PÉREZ, Rodrigo; DÍAZ, Rricardo; MEZA, Patricio; DONOSO, Sebastián. Análisis del mercado laboral docente: visión desde los sostenedores. Talca: Universidad de Talca, Instituto de Investigación y Desarrollo Educacional, Programa de Magíster en Política y Gestión Educacional, 2006. 55 p.

RAMA, Germán; NAVARRO, Juan Carlos. Carrera de los maestros en América latina. In: BID/PREAL. Maestros en América Latina: nuevas perspectivas sobre su formación y desempeño. Santiago de Chile: San Marino, 2004. p. 287-232.

SAN MIGUEL, Javier. Programa de Educación Básica rural. In: GARCÍA HUIDOBRO, Juan Eduardo (Ed.). La reforma educativa chilena. Madrid: Popular, 1999. p. 91-108.

SCHULMEYER, Alejandra. Estado actual de la evaluación docente en trece países de América Latina. In: PREAL-BID. Maestros en América Latina: nuevas perspectivas sobre su formación y desempeño. Santiago de Chile: San Marino, 2004. p. 25-66.

RUFINELLI, Ana; SEPÚLVEDA, Luis. Sistematización de la oferta de programas especiales de pedagogía en educación básica de las instituciones de educación superior chilenas. Santiago de Chile: Universidad Alberto Hurtado, 2005. 50 p.

SOTOMAYOR, Carmen. Capítulo 3. Programa de mejoramiento de la calidad de escuelas básicas de sectores pobres (p-900). In: GARCÍA HUIDOBRO, Juan Eduardo (Ed.). La reforma educativa chilena. Madrid: Popular, 1999. p. 69-90.
TEDESCO, Juan Carlos. Reformas educativas en América Latina: discusiones sobre equidad, mercado y políticas públicas. Estudios, Talca: Universidad de Talca, Instituto de Investigación y Desarrollo Educacional, 42 p., n. 14, 1998.

UNDURRAGA, Gonzalo. Capítulo 11. Programa de Becas al Exterior para profesores en servicio. In: GARCÍA HUIDOBRO, Juan Eduardo (Ed.). La reforma educativa chilena. Madrid: Popular, 1999. p. 215-231

VAILLANT, Denisse. Construcción de la profesión docente en América Latina. Tendencias, temas y debates. PREAL, Doc. de Trabajo n. 31, 2004.

VIAL, Gonzalo. Historia de Chile. Tomo I. La sociedad chilena en el cambio de siglo (1891-1920). Santiago de Chile: Santillana, 1987.

SEBASTIÁN DONOSO DÍAZ, doctor en educación por la Universidad Academia de Humanismo Cristiano (Chile), es director del Instituto de Investigación y Desarrollo Educacional de la Universidad de Talca. Sus publicaciones recientes son: "Financiamiento y gestión de la educación pública chilena de los años 90" (Cadernos de Pesquisa, v. 36, n. 127, p. 151-172, enero/marzo 2006); en coautoría con Víctor Cancino: "Desafios-chave do fortalecimento da gestão dos governos subnacionais chilenos da educação" (Ensaio, v. 14, n. 52, p. 311-339, jul./sept. 2006); en co-autoría con Miguel Bustamante: "Percepción de los docentes chilenos acerca del programa de escuelas focalizadas: elementos para el análisis de una política educativa de "acción positiva"” (Educação e Pesquisa, v. 32, n. 2, p. 293-310, mayo/ago. 2006). Investigación actual: "Nuevas estructuras políticas y de gestión de los gobiernos subnacionales em educacion”.E-mail: sdonoso@utalca.cl

Recebido em maio de 2007 Aprovado em maio de 2008 


\section{Resumos/Abstracts/Resumens}

Raquel Goulart Barreto

Roberto Leher

Do discurso e das condicionalidades

do Banco Mundial, a educação superior "emerge" terciária

$\mathrm{O}$ artigo discute as mudanças na educação superior produzidas pela recontextualização do discurso do Banco Mundial no Brasil, considerando os modos pelos quais as condicionalidades estabelecidas têm sido convertidas em práticas políticas. Está estruturado em duas partes. A primeira, centrada na materialidade do discurso, analisa as publicações que o banco identifica como chave para a educação superior, a partir das marcas constitutivas do "discurso das emergências". A segunda parte aborda a materialidade do novo imperialismo, explicita as contradições que o sustentam e focaliza as condições de produção do deslocamento do modelo universitário para o terciário.

Palavras-chave: discurso; novo imperialismo; recontextualização; educação superior
From the discourse and conditionalities of the World Bank, higher education "emerges" tertiary This article discusses changes in higher education produced by the recontextualization of the World Bank's discourse in Brazil, considering the ways in which "governance-related conditionalities" have been converted into political practices. It consists of two parts. The first one, centred on discourse as a material form of ideology, analyzes the "key publications" related to higher education, based on the features of what can be called a "discourse of emergences". The second part approaches the material dimension of the new imperialism, elicits its fundamental contradictions and focuses the conditions of production of shifting from the university-based model to the tertiary one.

Key words: discourse; new imperialism; recontextualization; higher education

Del discurso y de las condiciones del Banco Mundial, la educación superior "emerge" terciaria
El artículo discute los cambios en la educación superior producidos por la recontextualización del discurso del Banco Mundial en Brasil, considerando los modos por los cuales las condiciones establecidas han sido convertidas en prácticas políticas. Está estructurado en dos partes. La primera, centrada en la materialidad del discurso, analiza las publicaciones que el banco identifica como clave para la educación superior, a partir de las marcas designadas del "discurso de las emergencias". La segunda parte aborda la materialidad del nuevo imperialismo, explícita las contradicciones que lo sustentan y enfoca las condiciones de producción del dislocamiento del modelo universitario para el terciario.

Palabras clave: discurso; nuevo imperialismo; recontextualización; educación superior

Sebastián Donoso Díaz

$O$ aperfeiçamento docente no Chile (1990-2007): Estado versus mercado? 
A formação continuada dos docentes no Chile é uma área não regulada pelo Estado. Apesar de sua importância estratégica, reconhecida por todos os atores sociais políticos e institucionais, carece de uma política que ordene e estabeleça um marco regulatório para sua atuação. Esse fato é relevante pela deterioração da educação chilena e porque a reforma dos anos de 1990 parece não haver superado questões-chave nesse sentido; e também porque em suas raízes se encontram a reforma educativa de 1981, que alterou não somente aspectos básicos do sistema escolar, mas também a formação dos professores, cujas conseqüências estão sendo vislumbradas na atualidade. A crise é profunda e debate sobre seu futuro implica contrapor o Estado ao mercado, questão não considerada em alguns debates, mas parece que não há outra opcão senão enfrentá-la na atualidade.

\section{Teacher training in Chile (1990-} 2007): State versus market?

The in-service training of teachers in Chile is a deregulated area of the State, which in spite of its strategic importance, recognized by all social, political and institutional actors, has lacked a policy that orders and provides a regulatory framework in which to act. This phenomenon in Chile is of significant relevance for the general deterioration of education and because the reforms of the 1990's seem not to have solved key questions in this field. Secondly, because its roots are also to be found in the education reform of 1981, which changed not only basic aspects of the school system, but also the training of teachers, whose consequences are now being faced The crisis is deep and the debate on the future implies setting the State in opposition to the market, a question that is avoided in some debates, but for which apparently there is no other option except to face it.
Key words: in-service teacher training; teaching profession; teacher training in Chile

\section{El perfeccionamiento docente en Chile (1990-2007): ¿Estado versus mercado?}

La formación continua de los docentes en Chile es un área desregulada del Estado, la cual pese a su importancia estratégica, reconocida por todos los actores sociales políticos e institucionales, ha carecido de una política que ordene y provea un marco regulatorio para su actuar. Este fenómeno es de relevancia por el deterioro general de la educación y porque la reforma de los años de 1990 parece no haber superado cuestiones claves en este sentido; y también porque en sus raíces se encuentran la reforma educativa del año de 1981, que cambió no solamente aspectos básicos del sistema escolar, sino también la formación de los maestros, cuyas consecuencias se están vislumbrando en la actualidad. La crisis es profunda y su debate sobre el futuro implica contraponer el Estado al mercado, cuestión que se evade en algunos debates, pero pareciera que ya no hay otra opción que enfrentarla.

Palabras clave: formación continua de docentes; profesión docente; formación de docentes en Chile

Marcio da Costa

\section{Prestígio e hierarquia escolar -} estudo de caso sobre diferenças entre escolas em uma rede municipal

Este trabalho explora diferenças entre escolas públicas integrantes da mesma rede de ensino. É parte de um projeto de investigação que busca compreender os elementos que conferem diferenciação a estabelecimentos de ensino que, submetidos à mesma autoridade e às mesmas regras, integrando os mesmos sistemas e dispondo de recursos equivalentes, apresentam considerável disparidade em muitos aspectos e gozam de reputações claramente opostas. Nosso esforço, ancorado em literatura de pesquisa voltada à diferenciação do efeito das escolas (school matters, school effects) e à efetividade escolar (school effectiveness), compõe-se do estudo de caso em seis escolas municipais da cidade do Rio de Janeiro, organizadas em pares, em três diferentes regiões geográficas - e socioeconômicas. Cada par foi escolhido procurando contemplar escolas que usufruem prestígio claramente oposto, localizadas em áreas próximas, podendo atender a populações assemelhadas. Os resultados revelam um complexo sistema de hierarquias socioeconômicas, culturais e educacionais entrelaçadas.

Palavras-chave: prestígio escolar; hierarquias escolares; sociologia da educação

School prestige and hierarchy - a case study on differences between schools in a municipal network This paper explores the differences between public schools which are part of the same educational system. It is part of a research project that seeks to understand the elements which differentiate the quality of educational institutions which, when submitted to the same authority and to the same rules, and integrating the same system and having equivalent resources, present considerable disparity in many aspects and clearly have opposing reputations. Our endeavour, based on research literature directed at the differentiation between schools ("school matters" or "school effects") and school effectiveness, is composed of a case study in six municipal schools of the city of Rio de Janeiro, organized in pairs, in three different geographical and socioeconomic regions of the city. 